\title{
A 2.45 GHz Rectenna Screen-Printed on Polycotton for On-Body RF Power Transfer and Harvesting
}

\author{
Salah-Eddine Adami ${ }^{1}$, Dibin Zhu ${ }^{2}$, Yi Li ${ }^{2}$, Evangelos Mellios ${ }^{1}$, Bernard H. Stark ${ }^{1}$ and Steve Beeby ${ }^{2}$ \\ ${ }^{1}$ Faculty of Engineering, University of Bristol, Bristol, BS8 1UB, UK, salah.adami@bristol.ac.uk \\ ${ }^{2}$ Electronics and Computer Science, University of Southampton, Southampton, SO17 1BJ, UK
}

\begin{abstract}
This paper presents a 2.45 GHz rectenna on textile for RF power transfer and harvesting. Thin $350 \mu \mathrm{m}$ polycotton is used as a baseline flexible substrate and screen printing technique is used to map different layers. A $310 \mu \mathrm{m}$ interface layer is first printed on both sides of polycotton in order to smooth its surface. Silver layer is then printed onto the interface layers to map the antenna and the rectifier layouts. The substrate is characterized over the 0.5-4.5 GHz frequency range using the two-line method. A relative permittivity of 3.23 and a loss tangent of 0.06 have been obtained at $2.45 \mathrm{GHz}$. A standard patch antenna matched to $50 \Omega$ and a dual-polarization rectenna have been designed. Measurement of the return loss S11 of the patch antenna shows an excellent correlation with simulation results, thus indicating that the two-line characterisation is accurate. The performance of the polycotton antenna and rectenna is evaluated and compared with FR4. The measured radiation efficiency is $11 \%$ for the polycotton antenna, and $30 \%$ for the FR4 antenna. The polycotton rectenna generates $300 \mathrm{mV}$ and $100 \mu \mathrm{W}, 15 \mathrm{~cm}$ from a $100 \mathrm{~mW}$ EIRP transmitter.
\end{abstract}

Index Terms - wireless power transfer. RF energy harvesting, textile rectenna, textile antenna, polycotton.

\section{INTRODUCTION}

Wearable devices are increasingly being used by the public and in healthcare [1]. The energy source for wearables can be either a battery, an energy harvester [2] (e.g. photovoltaic cells, vibrational energy harvesters, thermoelectric harvesters), or wireless power transfer such as near-field inductive coupling or far-field RF. RF energy is a promising source especially with Internet-of-Things (IoT) emerging as a technology, where miniature receivers are required. In this case, antennas will not only be used for communication but also for the powering of low-power sensors.

This paper investigates the implementation of transmitting and receiving antennas on textile. The potential benefits to designers would be increased flexibility and comfort, and the ability to integrate large area antennas into garments, furniture, and other items in the user's environment.

Various flexible antenna based on textile have been presented so far [3]. Most of them are based on the use of commerciallyavailable conductive textile (or e-textile) associated with standard textile materials as a substrate. Where this method benefits from ease of prototyping, the different parameters and performance are not easily controllable. Also, the procedure for the realisation of a complete working system is not always outlined.
In this paper a patch antenna and a rectenna both fabricated using screen printing technique are presented and their performance evaluated experimentally. Section II presents the fabrication and characterisation of the polycotton substrate used. In Section III, the design of an antenna and a dualpolarisation rectenna (rectifying antenna) is presented. Finally Section IV present the experimental tests.

\section{FABRICATION PROCESS AND CHARACTERISATION}

\section{A. Fabrication Process}

The antenna was fabricated using screen printing. This technology was chosen because it is low cost, simple and suitable for patch fabrication. The fabric substrate was PES/cotton (65/35) which is widely used in the garment industry. As the fabric surface is rough and not suitable for direct deposition of functional layers, an interface layer needs to be printed on the fabric surface first, to reduce surface roughness, see Fig. 1. UV-curable ink Fabink-UK-IF1, developed by Smart Fabric Inks Ltd. [4] was used to print the interface layer.

Four deposits of interface ink were first made directly onto the fabric substrate and samples were cured under UV light for 30 seconds. After that, the printing continued with two deposits each time, followed by curing under the same conditions, until the target thickness of $310 \mu \mathrm{m}$ was achieved. Once printing on one side of the fabric substrate was finished, the sample was turned over and the same process was repeated until the target thickness $(310 \mu \mathrm{m})$ was achieved on this side as well.

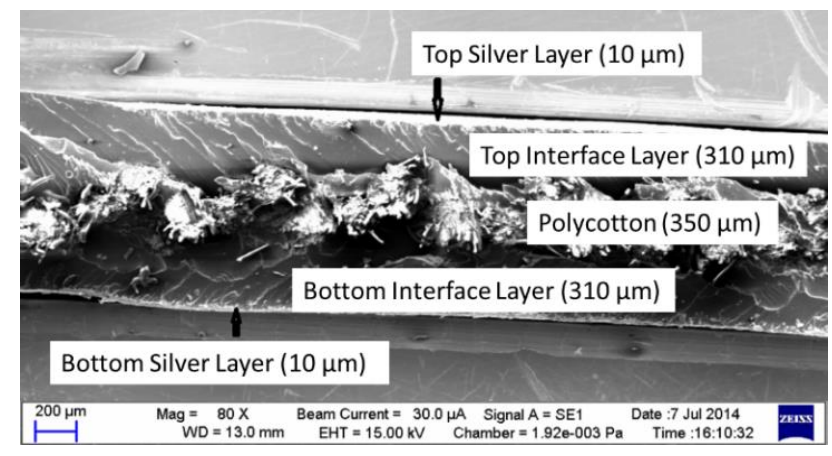

Fig. 1. SEM image (Scanning Electron Microscope) of the cross section of the screen-printed polycotton substrate. 
The conductive layers were printed using thermally curable silver polymer ink, Dupont 5000 [5]. One deposit was made for each conductive layer and samples were then cured at $120{ }^{\circ} \mathrm{C}$ for 10 minutes. The resulting thickness of the conductive layer is around $10 \mu \mathrm{m}$.

As silver polymer does not withstand high temperatures, silver epoxy was used to fix components and connectors, instead of soldering. Finally, the epoxy was cured at $100{ }^{\circ} \mathrm{C}$ for 15 minutes.

\section{B. Two-Line Method Characterisation}

In order to characterize the textile substrate over a wide frequency range, the two-line method is used [6], [7]. Relative permittivity $\varepsilon_{r}$ and loss tangent tan $\delta$ of the polycotton substrate can be estimated from a full-2-ports $S$ parameters measurement of two microstrip lines of different length.

The values $3.23 \pm 0.06$ and $0.06 \pm 0.01$ was obtained for $\varepsilon_{r}$ and $\tan \delta$ at $2.45 \mathrm{GHz}$ respectively (Fig. 2). Polycotton loss tangent is almost 3 times more than a standard FR4 substrate ( $\tan \delta$ (FR4) $=0.0175$ ). Dielectric loss of the polycotton substrate is expected to be at least triple that of a standard FR4 substrate.

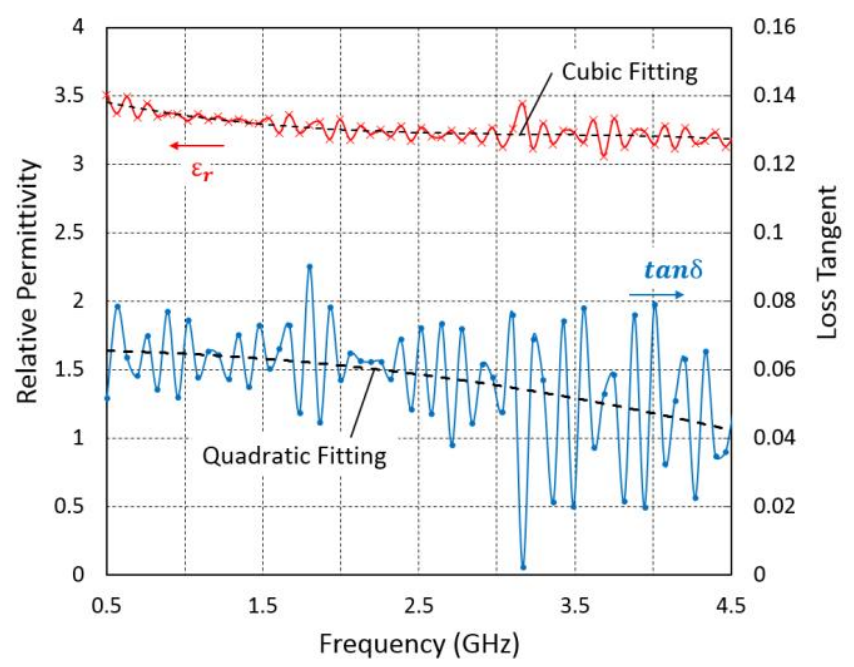

Fig. 2. Relative permittivity and loss tangent of the polycotton substrate measured using the two-line method.

\section{ANTENNA AND RECTENNA DESIGN}

\section{A. Patch Antenna Design}

Fig. 3 shows the textile patch antenna that has been designed for $\epsilon_{r}=3.23$ obtained in Section II, using the standard patch antenna design relationships [8]. A quarter-wave microstrip line matches the antenna to $50 \Omega$. A stub provides for postfabrication tuning. The fabricated antenna is shown in Fig. 4 (left).

\section{B. Dual-Polarisation Rectenna Design}

A dual-polarisation rectenna operates as two orthogonal antennas, and is therefore more suitable for wearable and moving applications than a single antenna with only one optimal direction. The rectenna is a square patch antenna, where two sides are connected to rectification circuits, see Fig. 4 (right). The antenna is designed to match directly to the complex impedance of the rectifier at the working frequency.

The RF/DC rectifier is a Schottky diode-based single stage voltage doubler [9]. The rectifier, including its substrate layout, has been simulated using Keysight Momentum ADS with the LSSP (Large signal S parameters) module. The simulated input impedance of the rectifier at $2.45 \mathrm{GHz}$ and $-15 \mathrm{dBm}(100 \mathrm{~mW})$ input power is $Z_{\text {rect }}=16-j 48$. The antenna edge length $L_{s q}$ has been reduced from its nominal half-wave length in order to compensate for the capacitive part of the rectifier. That way a conjugate matching has been obtained with $L_{s q}=32.95 \mathrm{~mm}$.

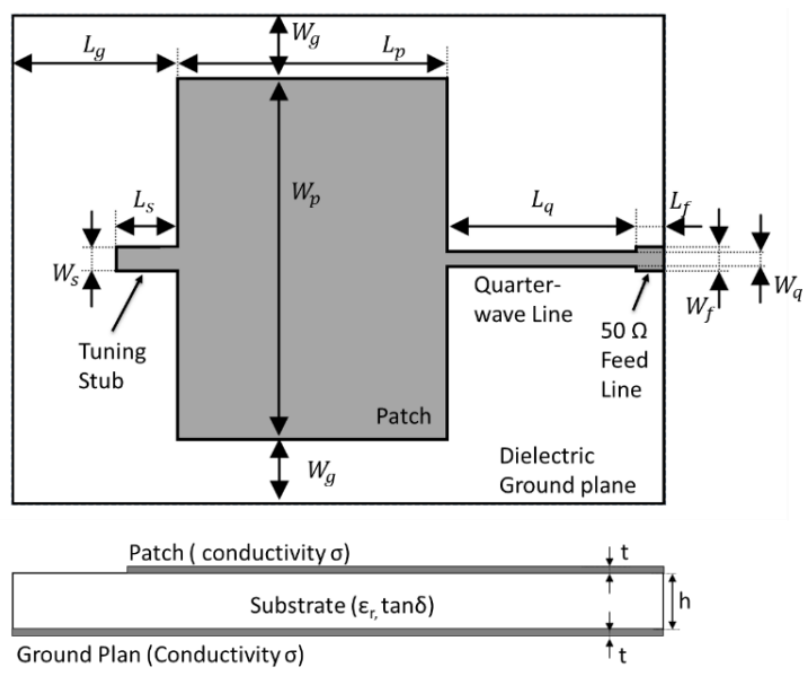

Fig. 3. Patch antenna design for $2.45 \mathrm{GHz}$, with quarter-wave microstrip line matching and tuning stub. Substrate: $\varepsilon_{r}=3.23$. Silver layer: Resistivity (from silver manufacturer datasheet) $=15 \mathrm{~m} \Omega$ / $s q, t=10 \mu \mathrm{m}$. Dimensions in $\mathrm{mm}: L_{p}=33.1, W_{p}=42.1, L_{g}=$ $15, W_{g}=10, L_{q}=17, W_{q}=1.25, L_{f}=5, W_{f}=2.24, L_{s}=5, W_{s}=$ 2.
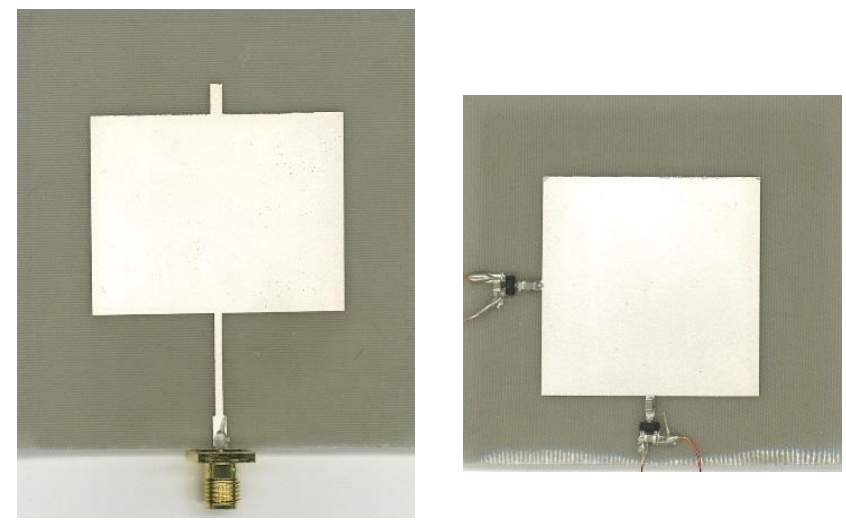

Fig. 4. Polycotton screen-printed prototypes: left, a $50 \Omega$ patch antenna, and right, a dual-polarisation rectenna. 


\section{EXPERIMENTAL TESTS}

\section{A. Experimental Tests of the Patch Antenna}

The measured return loss S11 for three identical designs of the textile patch antenna is shown in Fig. 5. All three antennas have a peak radiation frequency of $2.45 \mathrm{GHz}$, with similar return losses of $-11 \mathrm{~dB},-10.1 \mathrm{~dB}$ and $-9.7 \mathrm{~dB}$. We infer that remaining fabrication tolerances have little effect on the performance, and that the two-line characterisation has provided an accurate and reliable value for the relative permittivity $\varepsilon_{r}$.

The effect of the human body is also investigated by placing the antenna on different parts of the body: wrist, chest, leg. When the ground plan is oriented in the direction of tissues, no variation in return loss $\mathrm{S} 11$ is observed.

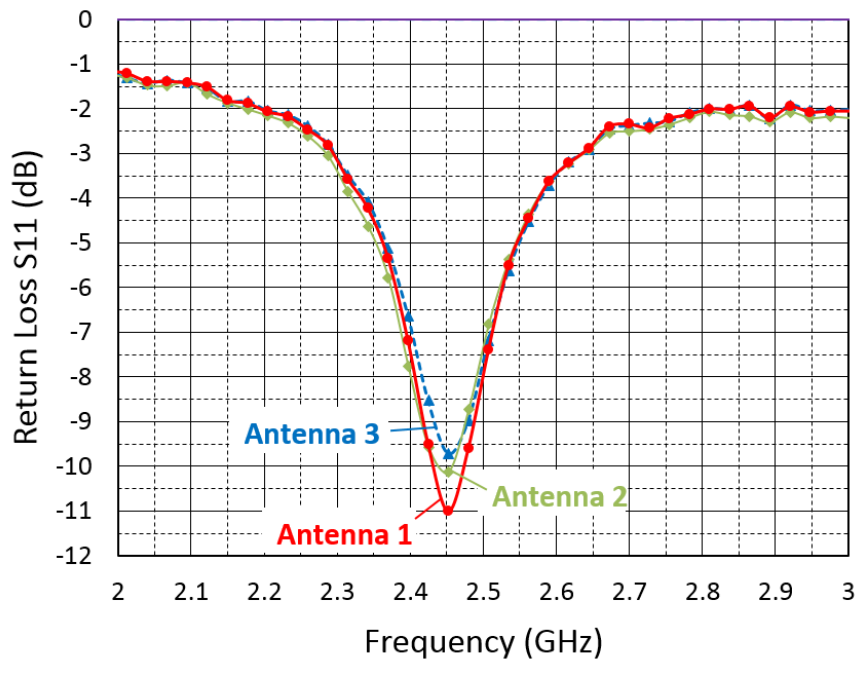

Fig. 5. Measured S11 parameter for three identical samples of the 50 $\Omega$ polycotton antenna.

The overall efficiency of an antenna depends not only on the mismatch losses but also on the conduction losses, and the dielectric losses within the substrate material. These losses are accounted for here by directly measuring the overall efficiency of an antenna in an anechoic chamber, as opposed to deriving it from the ratio of gain and directivity. The efficiency measurement is frequency dependent and is based on the comparison of the total radiated power (integration of the electric field over full 3D space) and the mismatch losses of the Antenna Under Test (AUT) with the same parameters of a highly efficient reference (REF) antenna, as shown in equation (1).

$$
\eta(f)=\left[\frac{\oiint\left|E_{A U T}(f)\right|^{2} d \Omega}{\oiint\left|E_{R E F}(f)\right|^{2} d \Omega}\right]\left[\frac{1-\left|S_{11, R E F}(f)\right|^{2}}{1-\left|S_{11, A U T}(f)\right|^{2}}\right]
$$

Where $f$ is the frequency, $\eta(f)$ is the frequency-dependent efficiency of the AUT relative to the reference antenna,
$S_{11, R E F}(f)$ and $S_{11, A U T}(f)$ are the return losses of the reference antenna and the AUT respectively, $E_{R E F}(f)$ and $E_{A U T}(f)$ represent the electric field total power radiation pattern for the reference antenna and the AUT respectively (in the far-field region), and $\Omega$ is the solid angle. The radiation patterns are found from full $3 \mathrm{D}$ pattern measurements using common cabling. The only compensation that needs to be applied in this method is for triple-travel along cables linking the test equipment to transmit and receive antennas in the anechoic chamber.

The method described above gives accurate relative efficiency figures and hence a highly efficient antenna needs to be used as the reference to yield an absolute value for efficiency. The reference used here is a simple tuned patch element with air as dielectric (hence no dielectric loss) on a large ground plane. Another option, that provides a similar accuracy, would have been to use a tuned monopole antenna. This efficiency estimation has an accuracy of around 5-10\%, which depends on the number of repeated measurements at $2.45 \mathrm{GHz}$.

The resulting measured efficiency is $11 \%$ for the polycottonbased antenna, and $30 \%$ for a $50 \Omega$ FR4 antenna. Dielectric loss of the textile substrate (polycotton + interface layers) is the dominant factor in the degradation of efficiency $(\tan \delta$ $($ polycotton $)=0.06$ and tan $\delta(F R 4)=0.0175)$.

\section{B. Experimental Test of the Rectenna}

The polycotton dual-polarisation rectenna was tested and compared with a similar FR4 rectenna using the set shown in Fig. 7. Note that the rectennas are symmetric, and therefore only one output is tested here.

In the first instance, the source power is kept constant at $150 \mathrm{~mW} \mathrm{EIRP}^{1}$, the rectenna is located $15 \mathrm{~cm}$ away in the line of sight, and its resistive load is swept over a wide range of values (Fig. 6). The optimal load $R_{\text {rect }}$ to maximize the DC load power is found to be $1 \mathrm{k} \Omega$. Therefore a power management circuit that emulates this resistance would optimize the rectenna operation [9]. The output voltage is around $1 \mathrm{~V}$ when the load is $50 \mathrm{k} \Omega$. Under these conditions, the rectenna could be connected to a commercial low-power DC/DC converter [10].

To compare the performance of the polycotton and FR4 rectennas, the source power was varied, with the DC load fixed at the optimal load for each rectenna, i.e. $1 \mathrm{k} \Omega$ and $2 \mathrm{k} \Omega$ for polycotton and FR4 respectively (Fig. 7). With the highest source power considered here (100 mW EIRP), $100 \mu \mathrm{W}$ and $230 \mu \mathrm{W}$ DC power were obtained with polycotton and FR4 rectennas respectively. The polycotton rectenna achieves its limit (less than $1 \mu \mathrm{W}$ DC power) when the source power is less than $10 \mathrm{~mW}$ EIRP. These results seem to be coherent with the efficiency values found previously.

${ }^{1}$ EIRP: Equivalent Isotropic Radiated Power. 


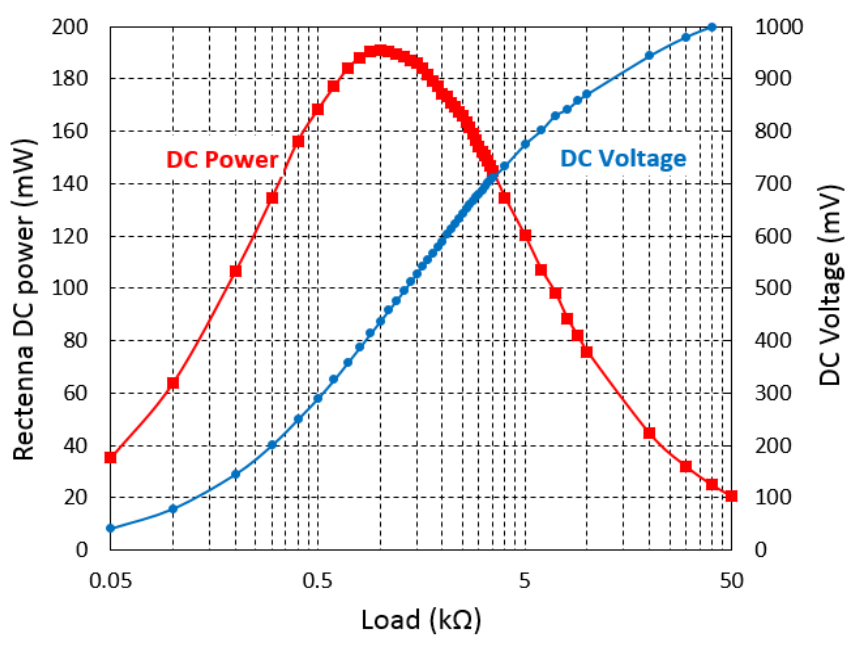

Fig. 6. Rectenna DC characteristics at $150 \mathrm{~mW}$ EIRP as function of the output load: DC power (red) and DC voltage (blue).

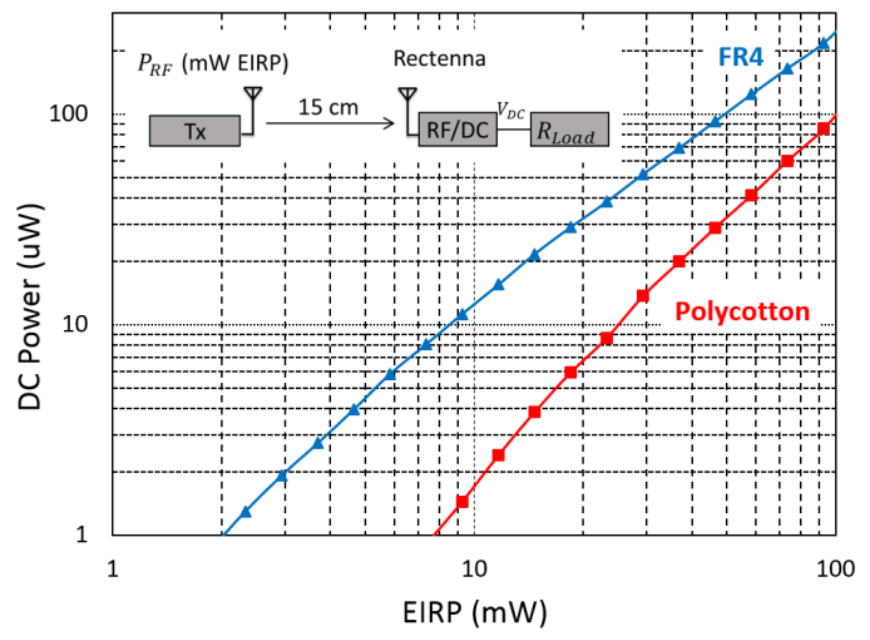

Fig. 7. Rectenna DC voltage at $15 \mathrm{~cm}$ from a $2.45 \mathrm{GHz}$ RF source as function of the source's Equivalent Isotropic Radiated Power (EIRP): Polycotton (red) and FR4 (blue).

\section{CONCLUSION}

This paper presents screen-printed patch antennas and rectennas for RF power transfer and harvesting on textiles. The planarised polycotton substrate has been accurately characterized using the two-line method. A $50 \Omega$ antenna and a dual-polarisation rectenna have been fabricated and tested experimentally. The performance of the realized substrate is around a third of standard FR4. In future work, improved textiles will be sought, to increase electrical performance, mechanical robustness, and resistance to chemicals.

\section{ACKNOWLEDGEMENT}

This work was performed under the SPHERE IRC funded by the UK Engineering and Physical Sciences Research Council (EPSRC), Grant EP/K031910/1.

\section{REFERENCES}

[1] M. M. Rodgers, V. M. Pai, and R. S. Conroy, "Recent Advances in Wearable Sensors for Health Monitoring," IEEE Sens. J., vol. PP, no. 99, pp. 1-1, 2014.

[2] Steve Grady and Cymbet Corporation, "Powering Wearable Technology and Internet of Everything Devices," 2014. [Online]. Available: http://www.cymbet.com/.

[3] R. Salvado, C. Loss, R. Gonçalves, and P. Pinho, "Textile materials for the design of wearable antennas: a survey.," Sensors (Basel)., vol. 12, no. 11, pp. 15841-57, Jan. 2012.

[4] "Fabink-UK-IF1, Smart Fabric Inks Ltd." [Online]. Available: http://www.fabinks.com.

[5] "Dupont 5000, silver conductor." [Online]. Available: http://www.dupont.com/.

[6] F. Declercq, H. Rogier, and C. Hertleer, "Permittivity and Loss Tangent Characterization for Garment Antennas Based on a New Matrix-Pencil Two-Line Method," IEEE Trans. Antennas Propag., vol. 56, no. 8, pp. 2548-2554, Aug. 2008.

[7] Moon-Que Lee and Sangwook Nam, "An accurate broadband measurement of substrate dielectric constant," IEEE Microw. Guid. Wave Lett., vol. 6, no. 4, pp. 168-170, Apr. 1996.

[8] J. M. Laheurte, Compact Antennas for Wireless Communications and Terminals: Theory and Design. Wiley, 2011, pp. 15-23.

[9] S.-E. Adami, P. P. Proynov, B. H. Stark, G. S. Hilton, and I. J. Craddock, "Experimental Study of RF Energy Transfer System in Indoor Environment," J. Phys. Conf. Ser., vol. 557, no. 1, p. 012005, Nov. 2014.

[10] Silicon Labs, "TS3310, A True 150 nA Iq, 0.9-3.6 Vin, Selectable 1.8-5 vout Instant-ON Boost Converter," Datasheet, 2014. [Online]. Available: http://www.silabs.com/. 